\title{
La charte qualité de l'ASOM poursuit son déploiement
}

\section{Roxane Kübler ${ }^{a}$, Esther Kraft ${ }^{b}$, Christoph Bosshard}

a Responsable opérationnelle de I'Académie suisse pour la qualité en médecine (ASQM) de la FMH; ' ${ }^{\circ}$ cheffe de la division Données, démographie et qualité (DDQ) de la FMH; ${ }^{c}$ Dr méd., vice-président de la FMH, responsable du département Données, démographie et qualité

La charte qualité de l'ASQM fête ses deux ans. Depuis, elle a été ratifiée par 70 organisations médicales qui se sont engagées à respecter ses principes, et plusieurs stratégies et rapports qualité ont déjà vu le jour ou sont en cours d'élaboration. Son déploiement se poursuit ainsi sous les meilleurs auspices.

\section{Contexte}

L'activité médicale est basée sur la responsabilité du médecin face à ses patients. La qualité de sa prestation fait naturellement partie de son travail et bénéficie aussi bien au patient et à ses proches qu'au médecin. De ce fait, il faut garantir et encourager la démarche qualité sous tous ses aspects. C'est un point fondamental de l'identité médico-éthique de tout médecin, du Code de déontologie de la FMH et du droit suisse ${ }^{1}$.

La charte qualité de l'Académie suisse pour la qualité en médecine (ASQM) vise à concrétiser cet engagement commun en faveur de la qualité, et permet aux organisations médicales de rappeler conjointement leur adhésion aux principes fondamentaux de la qualité en matière de transparence, d'obligation et de durabilité. Elaborée par les délégués au Forum Qualité de l'ASQM et par leurs suppléants, la charte qualité de l'ASQM existe à présent depuis deux ans et a fêté cette année sa $70^{\mathrm{e}}$ signature. Nous saisissons l'occasion pour remercier toutes les organisations signataires de leur important investissement!

\section{Contenu}

La charte repose sur trois piliers:

- La transparence - rendre visibles les activités des médecins pour promouvoir la qualité en médecine et la qualité des prestations médicales.

- Le caractère obligatoire - les organisations médicales signataires développent une stratégie en faveur de la qualité et font régulièrement le point dans un rapport qualité.
- La durabilité - la stratégie en faveur de la qualité s'inscrit dans le long terme et garantit le développement de la qualité. La démarche qualité fait partie intégrante de la formation prégraduée, postgraduée et continue des médecins et respecte les dispositions légales notamment en matière de protection des données.

\section{Rapports et stratégies qualité}

Les organisations signataires ont déjà rédigé dix stratégies et six rapports qualité qu'elles ont envoyés à l'ASQM. Nous leur adressons nos plus vifs remerciements pour leur précieux travail!

L'ASQM soutient les organisations dans la mise en place de la charte et la rédaction des documents et se renseigne régulièrement sur le type d'aide dont elles ont besoin. D'après le dernier sondage au printemps 2018, d'autres stratégies et rapports sont en cours d'élaboration ou de planification pour 2018/19.

Vous pouvez consulter les stratégies et rapports déjà disponibles sur le site Internet de l'ASQM: www.asqm.ch $\rightarrow$ Charte qualité $\rightarrow$ Stratégies qualité www.asqm.ch $\rightarrow$ Charte qualité $\rightarrow$ Rapports qualité

\section{Entretiens}

Nous avons interrogé trois organisations ayant adhéré à la charte qualité et ayant déjà rédigé un rapport et/ou une stratégie: une organisation cantonale, une société de discipline médicale et une organisation faîtière.
Cf. art. 58, 59 et 59a LAMal et art. 77 OAMal. 
Société des médecins du canton de Zurich (AGZ) - Dr Roger Wanner, membre du Comité

\section{Quelle a été la motivation principale de votre société} à signer la charte qualité de l'ASQM?

La qualité est une tâche permanente et constitue le fondement des activités de l'AGZ. L'amélioration continue et le maintien de la qualité élevée des soins médicaux sont à la base de chaque projet et initiative de l'AGZ.

\section{Pour votre société, quel est l'enjeu principal} de la démarche en faveur de la qualité?

Pour une organisation cantonale comme l'AGZ, la qualité se définit à travers les aspects suivants: qualité de la prise en charge, et en particulier de la prise en charge ambulatoire universelle et adaptée aux besoins. Tout le défi de la démarche en faveur de la qualité consiste pour l'AGZ à exercer une influence directe, et pas seulement indirecte.

\section{Où voyez-vous les chances de la stratégie qualité?} Grâce à sa stratégie qualité, l'AGZ définit ses domaines d'action visant à améliorer les différents aspects de la qualité, par exemple améliorer la qualité des soins grâce à une bonne prise en charge ambulatoire, à des projets, à des campagnes et au travail politique, mais aussi en analysant les données statistiques et en anticipant l'évolution de ses membres. La stratégie qualité nous offre la possibilité de structurer notre réflexion sur les différents aspects de la qualité et de les avoir présents à l'esprit. Elle montre ainsi que la qualité joue un rôle important et qu'elle est prise en compte dans le travail de notre association.

\section{Société suisse de pathologie - Prof. Joachim} Diebold, membre du Comité / assurance qualité Quelle a été la motivation principale de votre société à signer la charte qualité de l'ASQM?
Notre vocation. Depuis sa fondation, la SSPath s'est donné pour mission de garantir que les pathologues de Suisse exercent au plus haut niveau en comparaison internationale. Nous possédons d'ailleurs une commission dédiée à l'assurance qualité depuis plus de 20 ans.

\section{Pour votre société, quel est l'enjeu principal de la démarche en faveur de la qualité?}

Faire preuve de bon sens. C'est-à-dire soutenir la motivation intrinsèque et l'éthique professionnelle des pathologues en tant que moteurs principaux de la qualité, qui vise en fin de compte toujours la sécurité des patients, et non les noyer sous de multiples réglementations.

\section{Où voyez-vous les chances de la stratégie qualité?}

Elle renforce la confiance. Dans son rapport annuel sur la qualité, la SSPath publie des chiffres-clés notamment sur les sessions de formation continue, les directives en matière de qualité et les accréditations d'instituts, dans le but de conforter la confiance légitime de nos collègues cliniciens et des patients dans la qualité élevée de notre travail.

Association suisse des médecins-assistant(e)s et chef(fe)s de clinique (ASMAC) - Marcel Marti, directeur adjoint / responsable politique et communication

Quelle a été la motivation principale de votre société à signer la charte qualité de l'ASQM?

La qualité est le fil conducteur de notre travail, que ce soit dans le domaine de la formation postgraduée, de l'admission à pratiquer, du respect des horaires de travail légaux ou de la réduction de la bureaucratie. En adhérant à la charte, nous rendons ainsi notre engagement visible vis-à-vis de l'extérieur.

\section{Pour votre société, quel est l'enjeu principal}

de la démarche en faveur de la qualité?

Nous nous battons pour de bonnes conditions de travail et contre les dysfonctionnements. La qualité fait naturellement partie de cette mission et n'est donc pas directement perceptible. Lorsque, par exemple, le temps de repos des médecins est respecté, alors cela a une influence très positive non seulement sur le bienêtre des médecins, mais aussi sur la sécurité des patients.

\section{Où voyez-vous les chances de la stratégie qualité?}

En élaborant une stratégie, les organisations sont amenées à se pencher en toute conscience sur la question de la qualité. Elles tracent une trajectoire claire en direction de leurs objectifs, qu'elles peuvent ainsi documenter, suivre et contrôler, conférant à la qualité une importance (encore) plus grande.

\section{Conclusion}

Ces trois entretiens mettent en lumière les différents enjeux et défis de la démarche en faveur de la qualité. Rédiger une stratégie ou un rapport qualité permet de mener une réflexion structurée et consciente sur cette démarche et de s'en inspirer pour exploiter les synergies et construire sur ce qui existe déjà, mais aussi pour rendre possibles les nouveaux développements. Le travail mené par les organisations médicales et les médecins doit être intégré au processus législatif sur la qualité et l'économicité (15.083). 